\title{
Development of Dismantling Tools for LW9-72.5 Circuit Breaker Closing Spring
}

\author{
Zhu Yuanda ${ }^{1, a}$, Zhang Su ${ }^{1, a}$, Zhu Ming ${ }^{2, b}$, Zhu Shaohong ${ }^{3, c}$, Gao Tiejun ${ }^{3, c}$ \\ ${ }^{1}$ Liaoning Skill Training Center \\ ${ }^{2}$ Benxi Power Supply Company, State Grid Liaoning Electric Power Supply Co., Ltd. \\ ${ }^{3}$ Tieling Power Supply Company, State Grid Liaoning Electric Power Supply Co., Ltd. \\ a 22181221@qq.com, bxwl_650928@126.com, ${ }^{\text {c }}$ 40136962@qq.com
}

Keywords: installation tool, closing spring, SF6

\begin{abstract}
The installation tool for SF6 circuit breaker closing spring is characterized by: diameter of the annular outer end cap is larger than that of the concentrically arranged annular inner end caps; there are multiple circumferentially evenly arranged support rods fixed between them; there are also many circumferentially evenly arranged bolts connected with around the outer end cap nuts.
\end{abstract}

\section{Introduction}

High voltage circuit breaker is an important switchgear for power system with the dual tasks of controlling and protecting circuitry. In the high-voltage circuit breaker, spring mechanism is commonly used and there are significant amounts and types of involved springs. Therefore, their reliability have a significant impact on the safe operation of equipment and power system. It has been found by surveys that there are occasionally breakdown problems in closing spring of spring mechanism in the selected substation's high-voltage circuit breaker. Besides improper use and maintenance, design methodology is also questionable. Conventionally, condition parameter is considered as a fixed value, which is not accordant with the actual situation and also is unable to solve the failure probability problem of mechanical parts during operation. This equipment is a special installation tool for closing spring of SF6 circuit breaker particularly for SF6 circuit breakers overhaul equipment field, which can reduce production costs, shorten production time, improve production efficiency and make is more convenient and safe to replace and install SF6 circuit breaker closing spring.

High voltage circuit breaker is an important switchgear for power system with the dual tasks of controlling and protecting circuitry. In the high-voltage circuit breaker, spring mechanism is commonly used and there are significant amounts and types of involved springs. Therefore, their reliability have a significant impact on the safe operation of equipment and power system.

\section{Features of SF6 Circuit Breaker}

1) Electron and SF6 molecules almost fully mixed in contacting and form mixed ions of weight, which is of value for deionization and extinguishing of remaining arc columns. SF6 is of great negative charge and its molecules can capture quickly free electrons and form negative ions, which is retardant in conduction and thus accelerates the recovery rate of dielectric strength between arcs. Therefore, it is of great arc extinguishing property. Under $1.01 \times 105 \mathrm{~Pa}$ pressure, arc performance of SF6 is 100 times that of air and will not go bad and can be reused.

2) SF6 gas is excellent in insulating and arc extinguishing and thus brings SF6 circuit breaker the following advantages: strong breaking capacity, higher suitable fracture voltage that allows multiple continuous breaking times, suitable for frequent operation, low noise, no fire danger and small mechanical wear, making it an excellent "no maintenance" circuit breaker. It is applied in high-voltage circuit more and more often.

3) Pure SF6 gas is a great arc extinguishing medium. If used in frequently operated low-voltage electrical equipment, metal vapor will react with SF6 gas decomposition due to the frequently 
operated electric arc function and combine to generate powders of great insulation effect (hydrofluoric acid salt, sulphur based acid salt, etc.). The powders deposit on the contact surface and corrode seriously the contact material and thus the contact resistance increase dramatically, making it unreliable for the functioning of sealed contact filled with SF6 gas. Therefore, it is unsuitable to use SF6 arc distinguishing media in frequently operated low-voltage electrical equipment.

SF6 gas is of great corrosion for aluminum and harmful for phenolic resin laminate and ceramic insulation due to the corrosive gas generated under high temperature in discharge. When SF6 accounts for $20 \%$ to $30 \%$ in the mixed gas of SF6 and N2, its dielectric strength is the same with that of fully charged SF6 and the corrosive effect reduces greatly. Therefore, considering that SF6 is frequently used along with N2, water content of SF6 gas in SF6 circuit breaker should follow strictly the regulation and not exceed the standards, or water would react with SF4 in arc decomposition and produce corrosive hydrofluoric acid (H $2 \mathrm{O}+\mathrm{SF} 4 \rightarrow \mathrm{SOF} 2+2 \mathrm{HF}$ ). moisture content that reaches saturation will condense on the surface of insulation part, making the insulation strength reduce significantly and even resulting in creeping discharge. It has been proved by operating experience and the above discussions that insulation structure in SF6 circuit breaker is small and if the water content is high, the insulation effect will reduce greatly and contact resistance will increase significantly, making damages or explosion accidents more easily during operation. Therefore, all manufacturing plants and operating departments require a strict sealing process and also provide that water content of SF6 gas shall not exceed the standard. Standard in China is that water content of SF6 gas should be less than 300ppm (volume ratio).

4) SF6 circuit breakers takes SF6 gas as extinguishing medium. Under normal circumstances, SF6 is a non-flammable, odorless, non-toxic and inert gas with a density about five times that of air. However, under the effect of electric arc, a small part of SF6 gas would be decomposed and generate some low toxic fluorides, such as SOF2, SF4, SOF4 and SO 2 F 2, etc., which will affect the health of the body and is of corrosion and deterioration effect on metal parts. Therefore, SF6 circuit breaker is generally equipped with suction device and the adsorbents are activated alumina, activated carbon and molecular sieve, etc. The adsorption device can completely adsorb toxic substances decomposed by SF4 gas at high temperatures.

\section{FAQ in Circuit Breaker}

In the assembly process of SF6 circuit breaker, closing spring should be installed after the spring mechanism is assembled. The current closing spring installation method is: install the vertical rod in the central bottom of spring sleeve, insert the closing spring inside the spring sleeve, place spring compression on the top of closing spring, pressing spring compression into the spring sleeve along with closing spring by compressing, then install nut when the rod come through the center hole of spring compression and fix them inside the spring sleeve. This method of installing closing spring by compression machine greatly increases production cost and it is required during installation to fix spring sleeve on the press fixture, resulting in long production time, low productivity and possibly damages on operators if the unstably fixed spring pop up due to uneven load during pressing.

Upon the above technical problems, this paper proposes an installation tool for closing spring of SF6 circuit breaker that can reduce production cost, shorten production time, improve production efficiency and ensure safety operation. Its specialty lies in that diameter of the annular outer end cap is larger than that of the concentrically arranged annular inner end caps; there are multiple circumferentially evenly arranged support rods fixed between them; there are also many circumferentially evenly arranged bolts connected with around the outer end cap nuts.

\section{Processing Method}

In the traditional calculation of strength, values of external load, parts' geometry and strength performance of materials, etc. are considered and processed as fixed values. In fact, many parts are 
affected by multiple factors as material, manufacturing process, measurement and test and the parameters are uncertain; but reliable design can make up this shortage and takes these parameters as random variables by certain distribution. After being calculated according to the theory of probability and statistics and strength calculation, failure probability of mechanical parts under certain design conditions would be obtained and thus reliability of the product can be obtained to make it more accurate for people to learn about the quality of products.

Adjust the geometrical size of the spring. Reliability of the spring depends mainly on the size of its shear stress; increasing diameter of the spring wire or reducing diameter of the spring can make the shear stress decrease and reliability of the spring increases. However, it should be noted that stiffness of the spring in spring operating mechanism has a great impact on its dynamic performance. Generally speaking, stiffness of the spring in spring operating mechanism has been optimized according to using requirements. Therefore, stiffness of the spring should be maintained when adjusting the geometrical size of the spring, and it should be achieved by combined adjustments. For example, increasing the diameter of the spring wire $d$ and at the same time increasing the effective spring turn $\mathrm{n}$; reducing the diameter of the spring $\mathrm{D}$ and at the same time increasing the effective spring turn.

In operation, the closing spring and spring compression should be first placed inside the spring sleeve of SF6 circuit breaker, then infix the bolt at the edge of outer end cap into the corresponding bolt holes on the spring sleeve with nuts, place the inner end cap into the spring sleeve, tighten each nut evenly to make the installation tool for closing spring in SF6 circuit breaker towards the spring sleeve, use spring compression to make the inner end cape compressed towards closing spring, tighten up the installation lock nuts when the central rod pierces the spring gland inside the spring sleeve and remove the installation tool of closing spring in SF6 circuit breaker. Since the spring compression can press the closing spring inside the spring sleeve, the closing spring has such features as simple structure, convenient to remove and reassemble, reducing production cost, improve operating efficiency, avoiding pop-up of closing spring under uneven load during installation and ensuring safety of operation. As shown in Fig. 1:

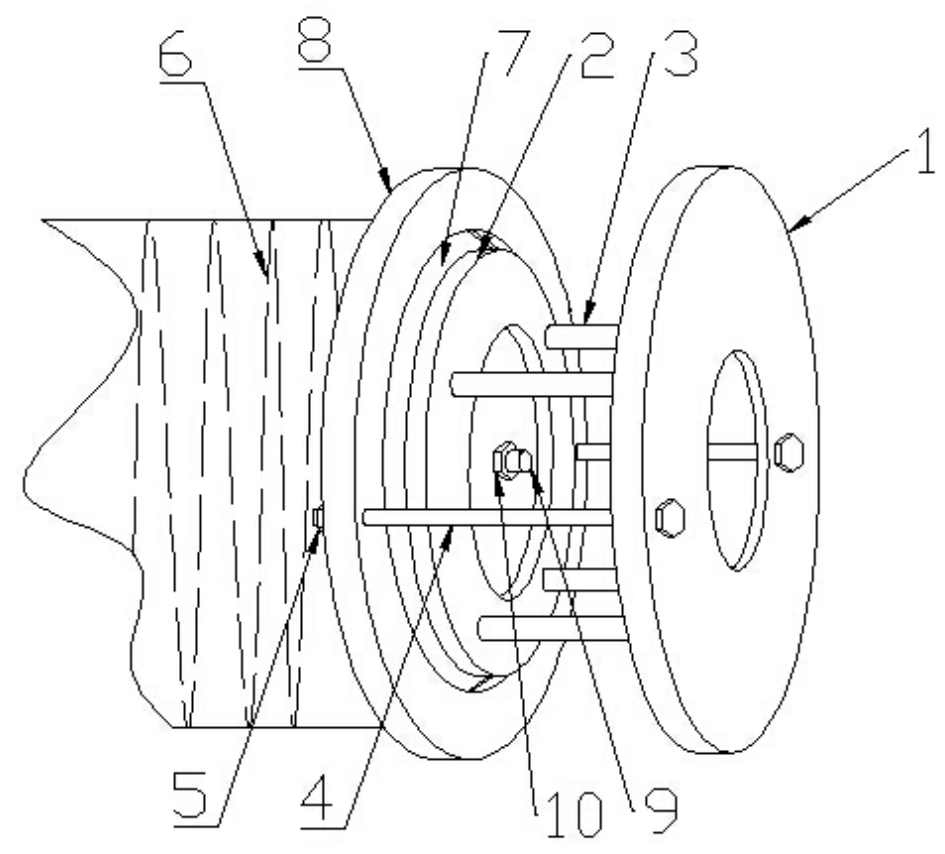

FIG 1: 1- outer end cape, 2-inner end cap, 3-rod, 4- bolt, 5-nut, 6- closing spring, 7- spring compression, 8spring sleeve, 9- rod, 10- lock nut. 


\section{Conclusion}

The special tools bear against the end cap of the front of the closing spring; then the screws that match the special tools are inserted into the bolt holes at the two end and fixed until the special tools are subject to stress. In the next step, the bolt in the front of connecting lever is dismantled by sleeve; after that, the bolts at the two ends of the special tool are also gradually loosened. The special tools are dismantled till the closing spring fully opens. Such steps can make the dismounting of closing spring and connecting level easier and save manpower and time. The most important is that the maintenance personnel are free from personal injury.

\section{References}

[1] Tan Rong, Jiang Qi. Design Book of Spring Operating Mechanism in High-voltage Vacuum Circuit Breaker. Gansu University of Technology, 2000.26 (1) 36-40.

[2] Ceng Xianghuang. Reliability Optimizing Design of Cylindrically Coiled Spring [J1. Machinery manufacturing and automation. 2005.34 (5): 12-15

[3] Wang Lianqing, Wang Erzhi. Analyzing High-voltage Electrons from Optimizing Design and Reliability of Spring Operating Mechanism in Vacuum Circuit Breaker. 2005,41 (3): 166 - L67.

[4] Guo Jing, Ma Zhiying, Lei Qing. Optimizing deign of Cam Mechanism in Spring Operating Mechanism of Vacuum Circuit Breaker II. High-voltage electrical appliances, 2004,40 (3): 420-423.

[5] Zhao Wei, Yang Mei, Liu Hongbin, Wu Wei, Research and development of GIS based on component. Computer and modernization 2005. 06

[6] Wang Ninghui Practical manual of electrical engineer (power supply), Beijing: Machinery Industry Press, 2006 